\title{
Marines fantômes. Aventures et mythologies de la mer chez Henri Bosco
}

Bruno Tritsmans

\section{(2) OpenEdition}

\section{Journals}

Édition électronique

URL : http://journals.openedition.org/studifrancesi/1209

DOI : 10.4000/studifrancesi. 1209

ISSN : 2421-5856

Éditeur

Rosenberg \& Sellier

\section{Édition imprimée}

Date de publication : 1 décembre 2015

Pagination : $538-545$

ISSN : 0039-2944

\section{Référence électronique}

Bruno Tritsmans, "Marines fantômes. Aventures et mythologies de la mer chez Henri Bosco », Studi Francesi [En ligne], 177 (LIX | III) | 2015, mis en ligne le 01 décembre 2016, consulté le 08 janvier 2021. URL : http://journals.openedition.org/studifrancesi/1209; DOI : https://doi.org/10.4000/studifrancesi. 1209

\section{(c) $(7)(9$}

Studi Francesi è distribuita con Licenza Creative Commons Attribuzione - Non commerciale - Non opere derivate 4.0 Internazionale. 


\title{
Marines fantômes. Aventures et mythologies de la mer chez Henri Bosco
}

\begin{abstract}
The interest for maritime adventure in literature, especially in the $19^{\text {th }}$ and $20^{\text {th }}$ century, finds its origin in the desire to oppose a society that is seen as «morass», and to valorize «vital energy» (Alain Corbin). This essay analyses the poetics of maritime adventure in the work of Henri Bosco (1888-1976). Henri Bosco, whose work is haunted in depth by the crisis of civilization (the «soleil noir»), projected to write a «roman marin», with the pirate Bras-de-fer as protagonist. In fact, he transforms this promethean figure in ferryman or phantom (Le Trestoulas (1934), L'Epervier (1963)), and opposes static figures such as the temple or the mas (L'Oustaou di Dieu) to the instable and sometimes threatening maritime theme. Those scriptural processes inform the poetic complexity of L'Enfant et la rivière (1945), very close to what can be called an art of mètis (Marcel Détienne, Jean-Pierre Vernant).
\end{abstract}

\section{Aventures marines, feux et cendres}

L'espace marin a exercé une fascination durable sur la littérature du $\mathrm{xx}^{\mathrm{e}}$ siècle, et ce pour les raisons qu'Alain Corbin a évoquées dans Le territoire du vide. L'imaginaire marin s'enracine en effet profondément dans une opposition avec la société. Alors que celle-ci est perçue comme aliénante, comme un «miasme», la mer offre l'occasion d'un regain d'énergie vitale, d'une confrontation virile avec la violence des éléments naturels ${ }^{1}$. La mer devient dès lors le théâtre d'une quête, voire d'une «mystique moderne» ou d'un «réenchantement du monde» ${ }^{2}$, et derrière l'aventure marine se profile l'ombre de Prométhée ${ }^{3}$.

En même temps, l'aventure marine tend, tout au long du $x x^{e}$ siècle, à se dégrader, et la littérature n'en garde alors, selon le mot de Jean-Yves Tadié, que le «goût de cendre» ${ }^{4}$. Il rejoint ainsi la réflexion plus générale sur l'aventure qu’ont menée Georg Simmel et Vladimir Jankélévitch, très sensibles à la précarité de l'aventure qui risque à tout moment de verser dans le jeu'. Le topos de l'aventure marine comme quête se fragilise, et la hiérophanie qui la sous-tendait devient «cryptique» ${ }^{6}$, au point de n'être

(1) A. Conbin, Le territoire du vide. L'Occident et le désir du rivage, Paris, Aubier, 1988, p. 319. Voir encore M. Roux, L'Imaginaire marin des Français, Paris, L'Harmattan, 1997, qui parle de «réponse à l'absurde» (p. 20).

(2) Ces expressions sont empruntées à S. VENAYRE, La gloire de l'aventure. Genèse d'une mystique moderne, Paris, Aubier, 2002, et à J. GRIFFET, Aventures marines. Images et pratiques, Paris, L'Harmattan, 1995.

(3) Sur le mythe de Prométhée dans l'imaginaire de la modernité, voir F. Flahault, Le crépuscule de Prométhée. Contribution à une histoire de la démesure bumaine, Paris, Mille et une nuits, 2008.
(4) J.-Y. Tadié, Le roman d'aventures, Paris, Puf, 1996 («Quadrige»), p. 189. Voir aussi L. Rasson et B. Tritsmans (éd.), Marines écrites. Récits de mer au $X X^{\mathrm{e}}$ siècle, Lille, Roman 20-50, 2004 («Actes»).

(5) Voir, de G. Simmel, «La philosophie de l'aventure», Mélanges de philosophie retativiste. Contribution à la culture philosophique, Paris, Alcan, 1912, pp. 110-116, et de V. JANKÉLÉVITCH, L'Aventure, l'ennui, le sérieux, Paris, Aubier, 1963.

(6) M. Eliade, Traité d'bistoires des religions, Paris, Payot, 1949, p. 3; Images et symboles, Paris, Gallimard, 1952, p. 25. 
plus lisible que sous la forme de traces, de résidus, et qui s'exprime par une (petite) mythologie du rivage. Le récit devient alors un palimpseste, où les topoi mythiques restent certes lisibles, mais disséminés et devenus fantômes textuels ${ }^{7}$.

\section{Le corsaire et ses doubles}

Telle est la perspective dans laquelle nous proposons de relire l'œuvre quelque peu oubliée de Henri Bosco (1888-1976), alors que bien des maîtres de la critique, de Gaston Bachelard à Jean-Pierre Richard, la tenaient en haute estime. Elle présente une sensibilité très forte à la «sauvagerie» ou au «mal» dans le monde, à la terre «qui s'enfonce» et qui donne un «sentiment de panique» ${ }^{8}$, - autant de figures de la barbarie de la guerre qu'il voit se profiler dès les années Trente, et, plus généralement, de la crise de la civilisation qui le hantait comme un «soleil noir» ${ }^{9}$. En même temps, il relègue cette sauvagerie dans les marges de l'œuvre, comme la «Préface» tardive (1957) du Mas Théotime $(1945)^{10}$, et il s'inscrit résolument dans une démarche réparatrice, voire rédemptrice. C'est sur ce contexte que la thématique marine vient se greffer sous des formes très diverses.

Henri Bosco affichait volontiers son goût pour le roman maritime, et notamment pour Conrad, Kipling et Melville ${ }^{11}$. Il se traduit par une prédilection pour la figure du corsaire Bras-de-fer ou Sabinus, qui figurait déjà dans une pièce d'Eglogues de la mer (1928), reprise dans Le roseau et la source (1949), ou encore dans Sites et mirages (1951). Il s'agit d'un guerrier qui combat les Turcs devant les îles Hyères, et Bosco se réfère souvent à ce personnage dans une perspective oppositionnelle. En 1935, il écrit à Gabriel Audisio qu'il veut se «corriger» des «fins de romans trop mystérieuses et incompréhensibles» ${ }^{12}$, et c'est plus précisément après «L'Habitant de Sivergues» (1933) et L'Ane culotte (1936) qu'il songe à écrire un «roman marin» ${ }^{13}$. On retrouve le personnage dans l'œuvre tardive, et notamment dans le cycle des Balesta, ou encore, dans Bras de fer, bref récit publié en 1959 dans la collection «Plaisir des contes».

En même temps, l'aventure du corsaire, qui relève de ce que Bosco a pu appeler son «désir profond», s'oriente dans un sens négatif et se transforme en naufrage: «ne pouvant pas naviguer un bateau, je l'ai fait couler» ${ }^{14}$.

(7) J. STAROBINSKI s'est montré attentif à la présence d'un «monde archaïque» dans les textes de la modernité (Les cheminées et les clochers, «Magazine littéraire», 280, (septembre 1990), pp. 2627). Dans la perspective de la poétique du récit, voir M. CHARLES, Introduction à l'étude des textes, Paris, Seuil, pp. 106 et 177 , et plus récemment, De la cobérence chez Balzac, «Poétique», 67, (2011), pp. 351-382.

(8) Henri Bosco et les voix de la terre, «Nouvelles littéraires», 2248, (22 octobre 1970), p. 2. Voir aussi J. Onimus, Le mal, «Cahiers Henri Bosco», 29, (1989), pp. 127-144.

(9) Voir la lettre à Jean Denoël du 2 avril 1974 («Cahiers Henri Bosco», 32-34, (1992-1994), p. 168), et à François Bonjean du 4 mai $1946($ («ahiers Henri Bosco», 37-38, (1997-1998), p. 138).

(10) Genèse du "Mas Théotime", «Cahiers Henri Bosco», 22, (1982), pp. 15-20.

(11) J.-P. Cauvin, Henri Bosco et la poétique du sacré, Paris, Klincksieck, 1974, p. 253; J. PrICE,
New light on the origins and symbolism of "Malicroix" by Henri Bosco, «The French Review», 47, (1974), p. 776.

(12) «Cahiers Henri Bosco», 39/40, (1999-2000), p. 19.

(13) Lettre à Noël Vesper du 28 janvier 1933 (Lettres à Noël Vesper, Terrasses de Lourmarin, 1986, p. 51. Voir aussi la lettre à sa mère du 17 mai 1936, citée par Claude Girault dans la très riche «note» qui suit «La Chanson du corsaire» («Cahiers Henri Bosco», 39-40, (1999-2000), p. 19).

(14) Propos cités par C. Girault, Propos sur "Malicroix", «Cahiers Henri Bosco», 19-20, (1980), p. 92, note 2. Voir encore, sur cette problématique, l'article de S. BECKETT, «Navires, naufrages et nautoniers/nautonières: le mythe de Charon dans l'œuvre de Henri Bosco», M. ZuPanCIC (éd.), Mythes dans la littérature contemporaine d'expression française, Ottawa, Le Nardis, 1994, pp. 171-180. 
Dans une œuvre fortement marquée par le jeu de miroir et le glissement de sens ${ }^{15}$, tout se passe comme si l'aventure marine et la figure du Corsaire se disséminaient. Je propose, dans cette perspective, de retracer un parcours du Trestoulas (1934) à L'Épervier (1963), pour focaliser ensuite sur le détail textuel dans L'Enfant et la rivière (1945).

\section{Barques-fantômes et tartanes}

Bosco a rédigé Le Trestoulas en mai-juin 1934, et il plaçait ce texte sous «l'inspiration de la Terre» ${ }^{16}$. Initialement, le récit est en effet profondément enraciné, et son protagoniste est un berger habitant un mas, la Jassine:

Mi-ferme, mi-bergerie, séparée du chemin par un jardin et une murette, la maison de Clapu était située assez haut dans les terres, à la racine des sources (pp. 20-21) ${ }^{17}$.

L'orientation verticale est encore renforcée par la «bergerie abandonnée» que Clapu possède sur le plateau du Trestoulas, couverte de «tuiles blanchâtres», et pardessus laquelle se dresse «une haute falaise» (p. 25). Mais la bergerie permet aussi d'accéder à la profondeur: elle s'enfonce «profondément dans la terre» (p. 37), et si on y trouve «un manteau de berger», celui-ci y côtoie «un paquet de bougies et une lanterne-tempête» (p. 114) destinées à éclairer le «trou béant» creusé derrière la cheminée, et qui permet d'accéder aux réserves aquatiques souterraines.

Sur le portrait de Clapu en berger se greffent des éléments menaçants, et le traditionnel bâton de berger devient ainsi une «grande pioche», dotée de «deux grandes dents luisantes» (p. 12). De même, le troupeau n'est mentionné qu'en passant (p. 87), et il cède la place au culte voué par Clapu au sanglier devant un «autel barbare» du monde aquatique souterrain (p. 118). Dans ce monde, Clapu se transforme de berger en passeur ${ }^{18}$ et il se meut sur un radeau à l'aide d'«une de ces pelles de bois qui [...] servent de van», et qui est éclairé par «un tronc de pin entier» (p. 117). Le Trestoulas présente ainsi à l'évidence une version nocturne du topos maritime, et l'espace aquatique devient lieu de sauvagerie et d'engloutissement ${ }^{19}$.

Il est cependant révélateur que la fin du récit réintroduise l'espace maritime. On y apprend en effet que l'histoire de Clapu est rapportée par André Cheynes à bord d'un «bateau ancré, bien à l'abri, dans la crique de la 'Maison de Dieu'» (p. 171).

Cette «Maison de Dieu» avait déjà été décrite dans un texte plus ancien intitulé L'Oustaou di Dieu, que Bosco avait envoyé à sa mère la veille de Noël 1936, et il s'agit en fait d'une bastide ruinée et abandonnée sur les falaises de Porquerolles. La ruine de la bâtisse est toutefois compensée par la remontée, dans le souvenir, d'un «vieux cantique de la mer»

(15) Voir le Diaire de Bosco, cité par J. Onimus: «À chaque personnage, je crée son ombre». Jean Onimus présente Bosco comme «emprisonné par ses propres reflets», qui inhibent l'action ( $\ll$ Le thème du double», Henri Bosco. Mystère et spiritualité, Paris, Corti, 1987, pp. 95-108). Voir encore les «Entretiens avec Monique Chabanne», «Cahiers Henri Bosco» 27, (1987), pp. 90, 106, 111, 131.

(16) Lettre à sa mère du 31 juillet 1934; publiée par C. GIRAult dans «Cahiers Henri Bosco» 39/40, (1999-2000), p. 24.
(17) Nos références renvoient à: H. Bosco, Le Trestoulas, Gallimard, 1935 («Folio»).

(18) Sur l'instabilité de la figure du berger chez Bosco, voir mon étude Le berger et ses fantômes. Fables en filigrane chez Henri Bosco, «Poétique», 174, (2013), pp. 251-260.

(19) Cf. encore la dérive en barque dans «Atlas» (Des Sables à la mer, Paris, Gallimard, 1950, pp. 169-189). 
où l'on voit trois tartanes bleues arriver d'Orient pour la fête des Rameaux avec un chargement de palmes ${ }^{20}$.

Bosco emprunte ce topos à l'un de ses textes les plus anciens et les plus fondamentaux. Parmi les Noëls et chansons de Lourmarin qu'il avait publiés en 1929 figure en effet une pièce intitulée «Le Noël de la barque», qui évoque «une barque chargée d'oranges» venue du «pays de l'Encens» ${ }^{21}$. Sites et mirages (1951) et Le Roseau et la source (1949) font d'ailleurs écho à ce topos fondateur, et évoquent à leur tour «des balancelles qui remontaient, chargées d'oranges, vers le Nord», et une «balancelle bleue» qui porte sur la mer «cent corbeilles d'oranges» ${ }^{22}$.

Dans Le Trestoulas, le topos s'infléchit de façon révélatrice:

Vers le large, par-delà l'île du Levant, une sorte de voilier-fantôme avait louvoyé jusqu'à la nuit. Maintenant il apparaissait, plus irréel encore, à quelques encablures de la côte (p. 171).

Aux «tartanes bleues» chargées de palmes du vieux cantique s'est substitué un «voilier-fantôme» sur fond nocturne. Du coup, le parcours rédempteur qui se dessinait dans Le Trestoulas entre le labyrinthe aquatique souterrain et la crique de la «Maison de Dieu» tend à se dissoudre, et tout se passe comme si le voilier-fantôme faisait écho à la barque infernale de Clapu. C'est pour pallier cette érosion du parcours rédempteur que le récit procède à une deuxième recontextualisation.

Plus de deux ans après avoir appris le récit dans la crique, le narrateur retrouve André Cheynes dans «une petite propriété» qu'il possède entre le cap Léoube et le cap Benat, «près de la mer» (p. 173). À la différence de la «Maison de Dieu», où les «tartanes bleues» deviennent «voilier-fantôme», cette propriété, située «près de la mer», offre une image de l'enracinement, qu'amplifiera plus tard Le Mas Théotime (1945), et la cueillette des amandes en est comme l'image emblématique ${ }^{23}$. Par cette poétique du récit feuilletée, Bosco tient manifestement à se distancier de ce qui lui paraissait des «créatures parties dans le monde des Ombres» ${ }^{24}$.

On retrouve ces éléments dans L'Épervier (1963), où Bosco évoque les derniers combats du corsaire dans les îles grecques. Après un long séjour à Pierrelousse, où ses ancêtres s'étaient retirés, le narrateur s'installe dans une maison «près du Cap Bénat», face à l'île du Levant (pp. 285-286) ${ }^{25}$. C'est de la pointe du cap, qui donne sur un rivage creusé de «petites criques» où les pêcheurs se mettent parfois à l'abri, qu'il observe «deux petites barques en train de pêcher» (p. 286), ou encore la sortie des bateaux à l'occasion d'une «nappe d'air qui venait du Sud» et qui apporte une «étrange douceur»:

l'on apercevait sur cette eau paisible leurs barques, qui posaient des palangres. Ou bien, à petits coups de rames, deux à deux, ils traînaient leurs filets sous l'île du Levant (p. 294).

(20) L'Oustaou di Dieu, «Cahiers Henri Bosco», 39/40, (1999-2000), pp. 26-30. Voir là encore la «note» très fine et documentée de Claude Girault.

(21) H. Bosco, Sept Noëls de Lourmarin, Paris, Editions M. Combre, pp. 8-9.

(22) Sites et mirages, Paris, Gallimard, 1951, p. 24; Le roseau et la source, Paris, Gallimard, 1949, p. 118.
(23) L'amande est symbole de «la naissance et de la résurrection» (C. MoREL, Dictionnaire des symboles, mythes et croyances, Paris, L'Archipel, 2002, p. 46).

(24) Dédicace (1973) du Trestoulas à Monique Baréa (fac-similé dans «Cahiers Henri Bosco», 41/42, (2001-2002), p. 127).

(25) Nos références renvoient à: H. Bosco, L'Épervier, Paris, Gallimard, 1963. 
Cette scène évoque les tartanes bleues du vieux cantique, mais comme en mineur: les barques traînent leurs filets, tandis que la référence à l'Orient se dissémine (le Sud, l'̂̀le du Levant).

La reprise en demi-teintes du topos rédempteur se poursuit encore. Le narrateur possède en effet encore une «bicoque» ou «un bout de bastide» situé au «sommet des falaises» de l'île du Levant, et qu'on appelle «L'oustaou dou marri-ferri» ou «La maison du mauvais garçon» (p. 295 $)^{26}$. En fait, le lieu s'avère habité par des inconnus qui y laissent des traces de foyer et un «petit calèn» (p. 297), et qu'un vieux pêcheur associe à la présence d'«une espèce de trois-mâts carré», «mâté et [...] caréné pour courir au vent comme un épervier de la mer» (p. 297). Plus tard, le pêcheur verra surgir un trois-mâts qui est une «ombre de navire» (p. 315), dont les feux clignotent sans qu'on ne voie un équipage. L'aventure héroïque du corsaire, qui avait vogué sur d'«immenses mers» (p. 318) avec «L'Épervier», s'infléchit dès lors dans le sens du fantomatique, et le narrateur même semble tenté de prendre le large sur le navire fantôme devenu menaçant (pp. 317-318) et de dériver ainsi vers les «profondeurs» (p. 315) $)^{27}$.

On retrouve ainsi les différentes composantes de la scène marine fondatrice de Bosco, mais le trajet rédempteur s'enlise. Même la «Maison de Dieu», qui était dans Le Trestoulas un refuge contre les fantasmagories, est doublée par la «maison du mauvais garçon», et est hantée par des figures spectrales.

\section{Retissages}

L'espace de L'Enfant et la rivière (1945) est réduit et bien différent des vastes étendues marines, car l'action se passe au bord du Rhône. Il est cependant révélateur qu'on y voie réapparaître la figure du navigateur, mais devenue cliché. Au début, il est thématisé par le biais d'un «vieux crocodile empaillé» (p. 16) ${ }^{28}$ qui a appartenu à l'oncle navigateur Hannibal, et qui est suspendu au plafond du grenier en compagnie d'un bric-à-brac de vieux habits, de chandeliers d'argent et de livres reliés de cuir qui gisent parmi des papiers jaunis (p. 16). Au moment du dénouement réapparaît un «ancien Navigateur», «gloire du village» (pp. 86, 95).

Â l'aventure marine fossilisée s'oppose le voyage de Pascalet dans les bras du Rhône. Il relate en effet qu'il a dérivé dans une vieille barque «un peu vermoulue» (p. 28) jusqu'à une île de bohémiens. Après y avoir libéré Gatso, il s'empare de la barque des bohémiens ornée d'«une rose des vents en cuivre» (p. 48) pour s'engager sur un «bras mort» (p. 49) de la rivière, qui forme une étendue lacustre que le protagoniste appelle le «monde des eaux dormantes» (p. 102).

L'aventure de Pascalet recompose l'aventure maritime, mais de façon en quelque sorte miniaturisée. On retrouve dans le récit une thématique diffuse de la menace et de l'engloutissement, qui se décline sur plusieurs plans ${ }^{29}$. D'entrée

(26) «La chanson du corsaire» que Bosco avait envoyée à sa mère en 1934 est signée «Lou marri-ferri» («Le mauvais garçon») («Cahiers Henri Bosco», 39-40, (1999-2000), p. 15), et parmi les manuscrits de Noëls et chansons de Lourmarin figure un «Noël du diable» («Cahiers Henri Bosco», 39-40, (1999-2000), pp. 12-14).

(27) Cette tentation est amplifiée dans Le Récif (1977), où le protagoniste, Markos, quitte le mas du Liguset pour Paros, puis pour un récif, et finit par être entraîné par un navire-fantôme. Voir aussi le «Liminaire pour L'Épervier», qui évoque une «côte abritée» hantée par d'《étonnantes épaves» («Cahiers Henri Bosco», 41-42, (2001-2002), p. 46).

(28) Nos références renvoient à: H. Bosco, L'Enfant et la rivière, Paris, Gallimard, 1953 («Folio/ Plus»).

(29) Voir, sur ce récit, les études d'A. DAvid ( $L e$ même et l'autre: jeux de miroirs dans "L'Enfant et la rivière", «Cahiers Henri Bosco», 30-31, (19901991), pp. 223-246) et de M. Arouimi (La quête my- 
de jeu, l'accès à la rivière est interdit au jeune Pascalet, parce qu'il y a «des trous morts où l'on se noie, des serpents parmi les roseaux» (p. 12), et certains poissons pêchés par Bargabot sont «hérissés d'épines», tandis que d'autres «ouvrent leurs gueules goulues dans le vide» (p. 22). La fin du récit mentionne encore le «monde mouvant et secret» (p. 111) des profondeurs aquatiques, où des monstres «aux écailles phosphorescentes» (p. 111) surgissent. Cette face nocturne du monde apparaît aussi sur l'île des bohémiens, où Pascalet voit une femme-sorcière égorger un coq sur le feu (p. 32), tandis qu'un ours rôde à proximité (p. 35). Enfin, au cour des «eaux dormantes», Pascalet et Gatso croient voir les traces des griffes d'une bête, qu'ils appellent «Racal» (p. 65), et qu'ils estiment d'une telle férocité «que même le taureau de combat et le buffle prennent la fuite devant lui» (p. 66). Tous ces éléments contribuent à évoquer le monde aquatique souterrain du Trestoulas, où Clapu vouait un culte au sanglier.

Face à cette menace de dérive dans la sauvagerie, L'Enfant et la rivière esquisse à son tour des parcours rédempteurs, lisibles jusque dans le détail textuel, et on retrouve là, de façon déplacée et indirecte, les tartanes bleues venues d'Orient et la «Maison de Dieu».

À l'eau noire, habitée par les monstres, s'oppose parfois une thématique solaire, en particulier quand, à l'aube ou au soleil couchant, «une buée d'or pâle se levait lentement de la lumière» (p. 44), ou que les eaux se colorent «de rose, d'or et d'hyacinthe» (p. 59). Le parcours rédempteur peut également se lire dans la présence d'oiseaux, tel ce «vol de plumes mouillées» (p. 44), et qu'on retrouve dans les autres figures du bestiaire ailé, tel le papillon (pp. 51, 59), la libellule ou le gerris (p. 59). De même, les étoiles formant de «grandes figures célestes» s'inscrivent sur cet axe qui oppose nuit et lumière:

Ils brûlaient très loin en silence. Leurs feux se reflétaient, en tremblant, dans la rivière, maintenant luisante et noire (p. 33).

Il arrive enfin que cette luminosité surgisse dans la profondeur des eaux, et sous les «reflets glauques», on voit alors se glisser un «doigt d'argent», puis «quelques bulles d'air, détachées d'une algue» (p. 51). Le monde aquatique peut devenir transparent, et les fonds «ri[ent] de soleil» (p. 112) et sont mystérieusement éclairés «en dessous par des feux invisibles» (p. 111). Sous le roc, entre les galets, quelquefois une «bulle d'air» (p. 112) éclot. Par ce réseau thématique subtil et complexe, le texte tisse ainsi des parcours rédempteurs fragiles et instables.

Par ailleurs, Bosco sollicite aussi des figures moins fluides, plus solides, à l'image de la «Maison de Dieu».

Au cœur des eaux dormantes se trouve en effet une île avec une chapelle, qui offre «un très vieux visage, roussi par les lichens et le long travail du soleil»:

Au-dessus de la porte, on avait creusé une niche où se trouvait une petite Vierge de plâtre colorié. Les couleurs en étaient parties. On devinait un peu de rose sur la robe. Une inscription en lettres bleues entourait cette modeste image (p. 69).

La chapelle de Notre-Dame-des-Eaux-Dormantes est corrélée à une thématique solaire, qu'on retrouve, affaiblie certes, dans le jeu des couleurs, et elle s'oppose à la

thique d'Henri Bosco dans "L'Enfant et la rivière", dans P. Schnyder (éd.), Métamorphoses du mythe. Réécritures anciennes et modernes des mythes an- tiques, Paris, L'Harmattan, 2008, pp. 617-629), qui lit l'Apocalypse, que Bosco avait traduite en 1942, en filigrane. 
dérive dans les eaux nocturnes. Tout se passe comme si l'on trouvait ici un écho de l'Orient et de ses «tartanes bleues».

Par ailleurs, ce lieu sacré discrètement rédempteur est ensuite relayé par le «petit théâtre» (p. 74) qui s'est installé dans le village, et de façon significative devant l'église $\mathrm{au}$ «porche profond et plein d'ombre» (p. 87). Le théâtre réintroduit la thématique rédemptrice par le biais de la «multitude de petits lampions et de grandes lanternes vénitiennes multicolores» (p. 87), ainsi que par la pièce de marionnettes jouée par Savinien et qui montre à la fois le vol d'un enfant par une sorcière et la réparation par saint Théotime. Tout se passe comme si l'espace théâtral, «filet de charme» d'un «magicien» (p. 92), permettait ainsi de conjurer la menace des eaux dormantes.

Après le spectacle, les lampions s'éteignent, mais il est révélateur qu'on ait oublié d'éteindre une chandelle, qui brûle

en tremblotant et la lueur de sa flamme invisible épandait au-dessus du léger toit une faible et mystérieuse couronne de lumière (p. 97).

Pascalet s'avoue fasciné par cette lumière fragile, mais la scène est interrompue par la venue de Bargabot. Celui-ci amène en effet «un petit bachot» avec «deux rames immenses et, comble d'arrogance, un mât» (p. 103), et sur lequel il invite Pascalet à «embarquer». Il hisse ensuite la toile «rapiécée» et appareille (p. 104), coupe les «tourbillons noirs» et saute «par-dessus les eaux tumultueuses» (p. 104). On voit que Bargabot reprend ainsi, avec ce radeau à la voile «rapiécée», le fil de l'aventure marine du corsaire, et ce d'autant plus qu'il possède une «immense canardière» qui laisse dans les airs «une longue traînée d'étincelles rougeâtres» (p. 104) ${ }^{30}$.

\section{Temples et nébuleuses}

Face à la dérive et à l'engloutissement qui menacent son imaginaire marin axée sur le corsaire, l'écriture de Bosco tend vers la complétude symbolique, vers ce que Paul Ricœur appelait les «grands symbolismes» ${ }^{31}$. C'est dans ce sens aussi qu'on peut lire la «Maison de Dieu», mais aussi, plus généralement, la fascination de Bosco pour «les temples au bord de la mer, puissamment assis sur la terre sombre» ${ }^{32}$, et dont le château de Lourmarin a sans doute été, à ses yeux, une des formes tangibles:

Je bâtis sans cesse au sein de mes nuées.

\section{Ou encore:}

Je construis des temples au milieu des nuages ${ }^{33}$.

(30) Dans Bargabot (1958) surgit à nouveau le souvenir du corsaire, sujet de «tout un livre à faire» (p. 72), mais Bargabot même, blessé par les nomades, meu rt en dérivant dans sa barque (p. 82).

(31) P. RICEUR, Le symbole donne à penser, «Esprit», 27, (juillet-août 1959), p. 71. Voir encore son entretien avec F. Ewald (Paul Ricoeur: un parcours philosophique, «Magazine littéraire», 390, (septembre 2000), p. 20 e.s.).

(32) Songe du temple, «Cahiers Henri Bosco», 8-9, (1975), pp. 3-4.

(33) Ces citations sont empruntées au Diaire de 1963 («Cahiers Henri Bosco», 39-40, (1999-2000), p. 36), et à R. YTier, Bosco, l'amour, la vie, Lyon, Aubanel, 1996, p. 190. L. MARCO (Quand les nuages ont un destin, «Cahiers Henri Bosco», 11, (avril-octobre 1976), pp. 72-75) a épinglé les mêmes images. Sur le château de Lourmarin, voir W.P. RomaIN (Henri Bosco à Lourmarin, «Cahiers Henri Bosco», 30-31, (1990-1991), pp. 167-192), et G. RIEGERT (De Naples à Lourmarin, une amitié méditerranéenne. Henri Bosco-Jean Grenier, dans G. Dugas (éd.), La Méditerranée, de Audisio à Roy, Paris, Manucius, 2008 pp. 49-55). Il est révélateur que Bosco ait refusé d'écrire sur le château: «Le château, c'est le château, il se suffit à lui-même» (R. YITIER, op. cit., p. 159). 
Cette poussée informe à la fois le trajet du Trestoulas à L'Epervier, mais aussi le détail de la poétique de L'Enfant et la rivière. On voit que la «maison de Dieu», appelée à conjurer la dérive mais réinvestie par le fantomatique, y prend des formes fragiles, tels la chapelle aux figures effacées et le théâtre de marionnettes ${ }^{34}$.

C'est à cette fragilisation de la complétude mythique que Maurice Blanchot s'était montré très sensible dans sa lecture de Hyacinthe ${ }^{35}$, et il est révélateur que Bosco ait à l'occasion évoqué une poétique de l'«à peu près» ${ }^{36}$, proche de cet art de la mètis cher à Dédale ${ }^{37}$, et dont le tissage des fils de nuages par le vent «à travers un treillis léger de vapeurs» ${ }^{38}$ offre une très belle image emblématique.

BRUNO TRITSMANS

(34) Il faut mentionner ici, en miroir, le double travail d'illustration de L'Enfant et la rivière par Georges Lemoine, qui cherche à conjurer la brisure par l'aquarelle (Gallimard, 1967), marquée par l'alliance entre les éléments et par une dynamique ascendante (oiseaux, papillons), ou par le dessin (Gallimard, 1979) et son jeu du microcosme et du macrocosme, du détail et de la totalité (le mur en pierres sèches, les galets de la rivière qui dessinent des figures,...). Voir sur ce point l'introduction de F. VIE à Georges Lemoine. La terre, l'eau, le ciel (Paris, Gallimard, 1987), et la belle étude d'I. Roussel-Gillet («Les petites musiques de
Georges Lemoine», Georges Lemoine, écrire, dessiner, Avranches, Editions H. de l'Abbaye, 2009, pp. 14-31).

(35) M. Blanchot, Faux pas, Gallimard, 1943, p. 221

(36) Pages du "Diaire", «Cahiers Henri Bosco», 39-40, (1999-2000), p. 32 .

(37) Sur Dédale et l'artisan, voir M. DÉTIENNE et J.-P. Vernant, Les Ruses de l'intelligence. La mètis des Grecs (Paris, Flammarion, 1974), et F. FronTisIDucroux, Dédale: mythologie de l'artisan en Grèce, Paris, Maspéro, 1975.

(38) L'Enfant et la rivière cit., p. 44. 